\title{
A Comparative Study of the Hydroxyl and Saponification Values of Polysorbate 60 in International Food Additive Specifications
}

\author{
Kazuaki Wakita ${ }^{1}$, Haruyoshi Kuwabara ${ }^{2}$, Noriko Furusho ${ }^{3}$, \\ Chiye Tatebe ${ }^{3}$, Kyoko Sato ${ }^{3}$, Hiroshi Akiyama ${ }^{3}$ \\ ${ }^{1}$ Oleo \& Specialty Chemicals Research Laboratory, NOF Corporation, Kawasaki-shi, Japan \\ ${ }^{2}$ Quality Assurance Department, Gunma Complex, Shin-Etsu Chemical Co. Ltd., Annaka-shi, Japan \\ ${ }^{3}$ Division of Food Additives, National Institute of Health Sciences, Tokyo, Japan \\ Email: akiyama@nihs.go.jp
}

Received November 30, 2013; revised January 4, 2014; accepted January 11, 2014

Copyright (c) 2014 Kazuaki Wakita et al. This is an open access article distributed under the Creative Commons Attribution License, which permits unrestricted use, distribution, and reproduction in any medium, provided the original work is properly cited. In accordance of the Creative Commons Attribution License all Copyrights (C) 2014 are reserved for SCIRP and the owner of the intellectual property Kazuaki Wakita et al. All Copyright (C) 2014 are guarded by law and by SCIRP as a guardian.

\section{ABSTRACT}

We investigated the hydroxyl and saponification values of 27 samples of Polysorbate 60 products that were commercially available worldwide. We observed that the values of most of the studied samples were not within the range established at the Joint FAO/WHO Expert Committee on Food Additives (JECFA), while they did agree with the specifications described in the USA, the EU and Japan. We believe that purities of the new commercial Polysorbate 60 samples are higher than those of the older products which were available when the JECFA specifications were discussed (around 1973). The present study suggests that the hydroxyl and saponification values of the current JECFA specifications for Polysorbate 60 should be re-evaluated.

\section{KEYWORDS}

Polysorbate 60; Polyoxyethylene Sorbitan Monostearate; Hydroxyl Value; Saponification Value; Emulsifier; Specification

\section{Introduction}

Polysorbate 60 (polyoxyethylene sorbitan monostearate or Tween 60) is a sorbitol-based nonionic surfactant which is commonly employed as emulsifier, dispersant, and stabilizer in a wide variety of food, cosmetic and pharmaceutical products [1]. The compound primarily consists of partial fatty acid esters such as stearate and palmitate, sorbitol-derived cyclic ethers (sorbitans and sorbides) polymerized with approximately 20 molecules of ethylene oxide per molecule of Tween (Figure 1) [2]. Polysorbate 60 is widely used in various food products, including confectionery items, bread, dressings and shortenings [3-5].

Polysorbate 60 was approved as a food additive in the USA in 1960 and in the EU in 1995, followed by South Korea, China, Taiwan, Canada, Australia, New Zealand and Japan. Although the specifications and standards for
Polysorbate 60 as a food additive have already been established in many countries, the ranges of hydroxyl and saponification values in the purity tests established at the $67^{\text {th }}$ Joint FAO/WHO Expert Committee on Food Additives (JECFA) are somewhat different from those established in the USA, the EU, Japan and so on (Table 1).

Table 1. JECFA specification deviation of Polysorbate 60 from EU, USA and Japanese in hydroxyl and saponification value.

\begin{tabular}{ccc}
\hline & Saponification value & Hydroxyl value \\
\hline JECFA & $41-52$ & $90-107$ \\
JAPAN & $45-55$ & $81-96$ \\
USA & $45-55$ & $81-96$ \\
EU & $45-55$ & $81-96$ \\
\hline
\end{tabular}




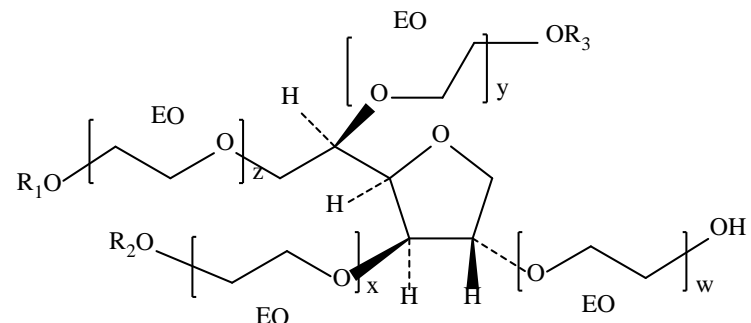

(a)

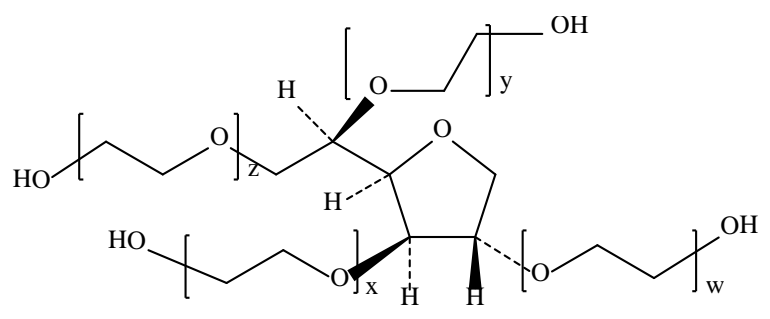

(b)

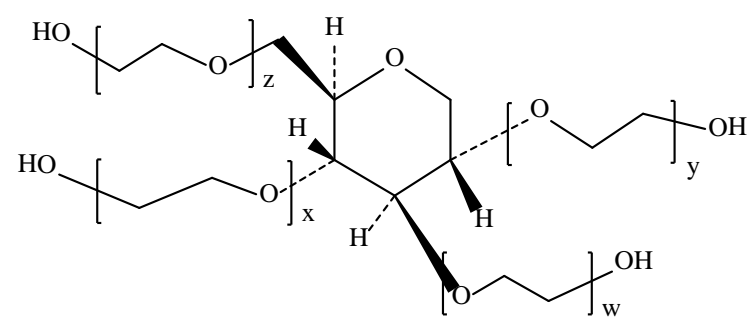

(c)

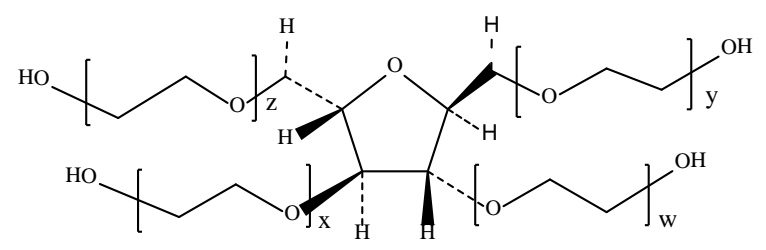

(d)

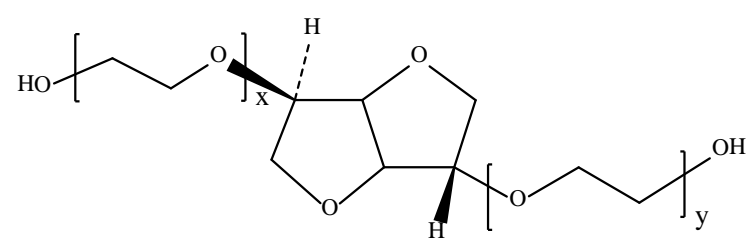

(e)

$\mathrm{EO}=$ oxyethylene group $\left(-\mathrm{CH}_{2} \mathrm{CH}_{2}-\mathrm{O}-\right) ; \mathrm{w}+\mathrm{x}+\mathrm{y}+\mathrm{z}=$ approx. 20; $\mathrm{R}_{1}=$ Stearic acid $\left(\mathrm{CH}_{3}\left(\mathrm{CH}_{2}\right)_{16} \mathrm{CO}-\right)$ or Palmitic acid $\left(\mathrm{CH}_{3}\left(\mathrm{CH}_{2}\right)_{14} \mathrm{CO}-\right)$; $\mathrm{R}_{2}=\mathrm{R}_{3}=\mathrm{H}$.

Figure 1. Typical structure of Polysorbate 60 (a) and sorbitol-derived cyclic ethers (b)-(e). (a) Polysorbate 60 (Polyoxyethylene sorbitan monostearate); (b) 1,4-sorbitan based polyethoxylate; (c) 1,5-sorbitan based polyethoxylate; (d) 1,4:3,6-isorbide based polyethoxylate; (e) 1,4:3,6-isorbide based polyethoxylate.

Since Polysorbate 60 is commercially available worldwide as a food additive, multiple different specifications for this compound cause many troubles and affect the fair trade between countries. In addition, they might induce serious trade disputes. Therefore, it is important to investigate whether the hydroxyl and saponification values of the commercially available Polysorbate 60 food additives are within the range specified by the JECFA.

In this study, we determined the hydroxyl and saponification values of the commercially available Polysorbate 60 products. We observed that the measured values were not within the range specified by the JECFA, although they did agree with the specifications prescribed in the USA, the EU and Japan. We discuss some of the factors responsible for these differences.

\section{Materials and Methods}

\subsection{Samples}

Twenty seven commercially available Polysorbate 60 products were provided by the Japanese, American, and European manufacturers (Table 2). The data reported for samples 7 - 9 can be considered as reference values because these are industrial-grade samples.

\subsection{Reagents and Chemicals}

The following chemicals were all extra pure grade (unless stated otherwise) and purchased from Kanto Chemical Co. Inc., Japan: pyridine, acetic anhydride, ethanol, $0.5 \mathrm{~mol} / \mathrm{L}$ ethanolic potassium hydroxide solution for the saponification analysis, phenolphthalein (10 g/L), diethyl ether, and $0.5 \mathrm{~mol} / \mathrm{L}$ hydrochloric acid as well as a 0.1 $\mathrm{mol} / \mathrm{L}$ potassium hydroxide solution for the volumetric analysis.

\subsection{Analysis of the Hydroxyl Value}

The hydroxyl value corresponds to the milligrams of potassium hydroxide $(\mathrm{KOH})$ required to neutralize an equivalent amount of acetic acid combined with hydroxyl groups in $1 \mathrm{~g}$ of a sample. The analysis was conducted in accordance with Japan's specifications and standards for food additives. The procedure is as follows:

The sample was carefully weighed (approximately $1 \mathrm{~g}$ ), placed in a $200 \mathrm{~mL}$ round-bottom flask, and mixed with $5 \mathrm{~mL}$ of the pyridine-acetic anhydride stock solution. A small funnel was placed at the neck of the flask and the temperature of the mixture was raised $\left(95^{\circ} \mathrm{C}-100^{\circ} \mathrm{C}\right)$ by using an oil bath (BK-33, HiTEC Corporation, Japan). After keeping the temperature constant for one hour, the flask was removed from the oil bath and the reaction mixture was cooled down to ambient temperature. Residual acetic anhydride was hydrolyzed to acetic acid by adding one milliliter of water. The reaction mixture was then reheated in the oil bath (for $10 \mathrm{~min}$ ), and after cooling, the funnel and the neck were washed down into the flask using $5 \mathrm{~mL}$ of neutralized ethanol. The resulting mixture was titrated against $0.5 \mathrm{~mol} / \mathrm{L}$ ethanolic $\mathrm{KOH}$ 
Table 2. Saponification and hydroxyl values in 27 commerical Polysorbate 60 products.

\begin{tabular}{|c|c|c|c|c|c|c|c|c|}
\hline \multirow{2}{*}{$\begin{array}{c}\text { No. } \\
1\end{array}$} & \multirow{2}{*}{ Manufacturers } & \multirow{2}{*}{ Nationalities } & \multicolumn{2}{|c|}{ Saponification value } & \multicolumn{2}{|c|}{ Hydroxy value } & \multicolumn{2}{|c|}{ Acid value } \\
\hline & & & & Ave. and S.D. & & Ave. and S.D. & & Ave. and S.D. \\
\hline 2 & \multirow[t]{2}{*}{ Company A } & \multirow[t]{2}{*}{ Japan } & 50.7 & \multirow[t]{2}{*}{$50.4 \pm 0.25$} & 98.5 & $97.3 \pm 1.01$ & 0.5 & \multirow[t]{2}{*}{$0.3 \pm 0.15$} \\
\hline 3 & & & 50.2 & & 96.7 & & 0.3 & \\
\hline 4 & \multirow{3}{*}{ Company B } & \multirow{3}{*}{ Japan } & 53.2 & \multirow{3}{*}{$52.8 \pm 0.40$} & 88.5 & & 0.4 & \multirow{3}{*}{$0.3 \pm 0.31$} \\
\hline 5 & & & 52.7 & & 90.1 & $89.8 \pm 1.22$ & 0.8 & \\
\hline 6 & & & 52.4 & & 90.9 & & 0.2 & \\
\hline $7^{* 1}$ & \multirow{8}{*}{ NOF } & \multirow{8}{*}{ Japan } & 52.5 & \multirow{8}{*}{$52.2 \pm 1.53$} & 79.3 & & 0.5 & \multirow{8}{*}{$0.3 \pm 0.20$} \\
\hline $9^{* 1}$ & & & 51.9 & & 76.6 & & 0.7 & \\
\hline 10 & & & 50.9 & & 81.5 & & 0.2 & \\
\hline 11 & & & 52.6 & & 87.7 & $83.8 \pm 5.91$ & 0.2 & \\
\hline 12 & & & 54.0 & & 91.0 & & 0.2 & \\
\hline 13 & & & 53.9 & & 92.0 & & 0.3 & \\
\hline 14 & & & 52.0 & & 85.0 & & 0.1 & \\
\hline 15 & & & 49.0 & & 83.0 & & 0.1 & \\
\hline 16 & \multirow[b]{2}{*}{ Company $C^{* 2}$} & \multirow[b]{2}{*}{ USA } & 51.6 & \multirow[b]{2}{*}{$51.6 \pm 0.40$} & 84.4 & & 0.7 & \multirow[b]{2}{*}{$0.7 \pm 0.25$} \\
\hline 17 & & & 52.0 & & 86.1 & $85.8 \pm 1.32$ & 1.0 & \\
\hline 19 & \multirow{3}{*}{ Company D } & \multirow{3}{*}{ Germany } & 52.2 & \multirow{3}{*}{$52.1 \pm 0.26$} & 90.5 & & 0.5 & \multirow{3}{*}{$0.6 \pm 0.10$} \\
\hline 20 & & & 52.3 & & 88.8 & $89.6 \pm 0.86$ & 0.7 & \\
\hline 21 & & & 51.8 & & 89.4 & & 0.6 & \\
\hline 22 & \multirow{6}{*}{ Company E } & \multirow{6}{*}{ UK } & 49.7 & \multirow{6}{*}{$49.7 \pm 0.72$} & 85.2 & & 0.3 & \multirow{6}{*}{$0.6 \pm 0.22$} \\
\hline 23 & & & 48.8 & & 88.9 & & 0.5 & \\
\hline 24 & & & 49.9 & & 83.9 & & 0.4 & \\
\hline 25 & & & 49.2 & & 83.0 & $85.4 \pm 2.02$ & 0.7 & \\
\hline 26 & & & 49.9 & & 85.8 & & 0.9 & \\
\hline 27 & & & 50.9 & & 85.5 & & 0.7 & \\
\hline
\end{tabular}

${ }^{* 1}$ Industrial grade; ${ }^{* 2}$ Company $\mathrm{C}$ now belongs to the other manufacturer.

using $1 \mathrm{~mL}$ of phenolphthalein as the indicator. The same procedure was followed without the sample (blank determination) and the hydroxyl value was calculated using Equation (1)

Hydroxyl value

$$
=\frac{(a-b) \times 28.05}{\text { Weight }(\mathrm{g}) \text { of the sample }}+\text { Acid value, }
$$

where $a$ and $b$ are the volumes $(\mathrm{mL})$ of ethanolic $\mathrm{KOH}$ consumed in the blank determination and in the titration of the sample, respectively.

\subsection{Analysis of the Saponification Value}

The saponification value corresponds to the milligrams of potassium hydroxide $(\mathrm{KOH})$ required to saponify the esters and neutralize the free acids in $1 \mathrm{~g}$ of a sample. The analysis was conducted in accordance with Japan's specifications and standards for food additives. The pro- 
cedure is as follows:

The sample was carefully weighed (approximately $2 \mathrm{~g}$ ), placed in a $200 \mathrm{~mL}$ flask, and mixed with $25 \mathrm{~mL}$ of 0.5 $\mathrm{mol} / \mathrm{L}$ ethanolic KOH. A short reflux condenser (Vidrex Co. Ltd., Japan) was connected to the neck of the flask, and the mixture was gently heated for one hour (under frequent stirring) using a water bath (IWB-250, AS ONE Corporation, Japan). After cooling, $1 \mathrm{~mL}$ of phenolphthalein was added to the reaction mixture. The residual $\mathrm{KOH}$ in the mixture was immediately titrated against 0.5 $\mathrm{mol} / \mathrm{L}$ hydrochloric acid. If the mixture was turbid, the titration was performed on warm samples (solution becomes clear when warmed). The same procedure was followed without the sample (blank determination), and the saponification value was calculated using Equation (2)

$$
\text { Saponification value }=\frac{(a-b) \times 28.05}{\text { Weight }(\mathrm{g}) \text { of the sample }},
$$

where $a$ and $b$ are the volumes (mL) of hydrochloric acid consumed in the blank determination and in the titration of the sample, respectively.

\subsection{Analysis of the Acid Value}

The acid value is the milligrams of potassium hydroxide $(\mathrm{KOH})$ required to neutralize the free acids in $1 \mathrm{~g}$ of a sample. The analysis was conducted in accordance with
Japan's specifications and standards for food additives. The procedure is as follows:

The sample $(10 \mathrm{~g})$ was weighted in a $250 \mathrm{~mL}$ glassstoppered flask. $100 \mathrm{~mL}$ of a mixture of diethyl ether and ethanol (95) (1:1 or 2:1) was added as the solvent, and if necessary, the sample was dissolved by warming. Then, a few drops of phenolphthalein were added and the solution was and titrated against $0.1 \mathrm{~mol} / \mathrm{L} \mathrm{KOH}$ (until the solution developed a light-red color which persisted for $30 \mathrm{~s}$ ). If the sample solutions were turbid at low temperatures, the titrations were performed on warm samples. The acid value was calculated using Equation (3)

Acid value

$$
=\frac{\text { Volume }(\mathrm{mL}) \text { of } 0.1 \mathrm{~mol} / \mathrm{L} \mathrm{KOH} \text { consumed } \times 5.611}{\text { Weight }(\mathrm{g}) \text { of the sample }}
$$

\section{Results and Discussion}

The hydroxyl and saponification values of several Polysorbate 60 samples are shown in Table 2 and Figure 2. The results indicate that the average hydroxyl values of all the samples are lower than the median value of the JECFA specification. Only samples 1, 2, 3, 5, 6, 12, and 19 are within the range specified by the JECFA (41 - 52). Except for samples 1 - 3, the hydroxyl values of all the samples were close to the lower limit (41) of the JECFA

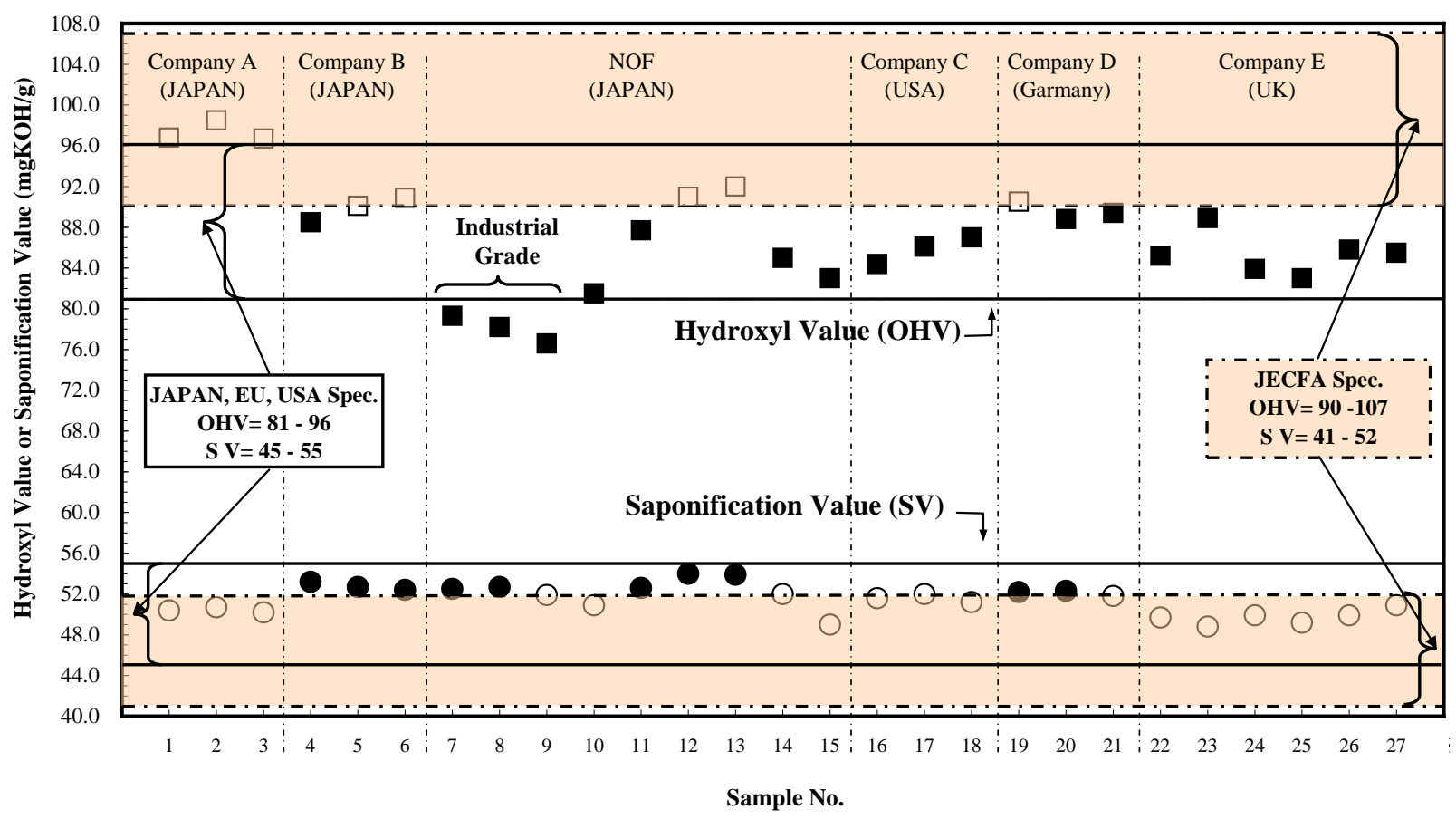

Figure 2. Plot of the saponification and hydroxyl values of 27 commercial Polysorbate 60 products. The shaded areas represent the JECFA spec range of hydroxyl value and saponification value. Squares $(\square$ or $\square)$ and circles $(\bullet$ or $\circ)$ represent saponification value and hydroxyl value, respectively. Closed circles $(\bullet)$ and squares $(\bullet)$ represent that they are out of the JECFA spec, and opened circles $(\circ)$ and squares $(\square)$ represent that they are within the JECFA spec. 
specification. All the samples (except for samples 1 - 3 of company A and samples 7 - 9, i.e., the industrial-grade probes) were within the range of the specification values accepted in the USA, the EU, and Japan (45 - 55). Since the hydroxyl value generally corresponds to the content of free hydroxyl groups in a compound, the hydroxyl values of Polysorbate 60 samples may give us an indication of the amount of free sorbitol-derived cyclic ethers present in those samples (Figure 1). This suggests that if the hydroxyl values in the samples are high, the total amount of free sorbitol-derived cyclic ethers as well as the acid values in the samples will also be comparably high. We showed that the average acid values determined for all the studied samples appear to be within the range (Not more than 2) specified by the JECFA (Table 2). The present results suggest that the commercial products available at the time when the JECFA specifications were established might contain higher amounts of free sorbitol-derived cyclic ethers compared with current commercial products.

Saponification values of samples 3 - 8, 11 - 13, 19, and 20 were within the range specified by the JECFA (90 107), whereas those of the remaining samples were not. However, the values of all the samples were within the range accepted in the USA, and the EU, and Japan (81 96). Since the saponification value generally indicates the content of ester groups in a compound, this value should decrease as the amount of free sorbitol-derived cyclic ethers increases in the Polysorbate 60 samples.

As shown in Table 1, the hydroxyl and saponification values of Polysorbate 60 specified by the JECFA are somewhat different from those accepted in several countries. Since impurities such as free sorbitol-derived cyclic ethers may raise the hydroxyl value and lower the saponification value of the samples, the two values measured for Polysorbate 60 will vary with the amount of impurities present in the samples. The hydroxyl value for Polysorbate 60 specified by the JECFA is higher than the values accepted in the USA, the EU, and Japan, whereas the JECFA-specified saponification value is lower than those accepted in the mentioned countries. The JECFA specifications for Polysorbate 60 were established at the $17^{\text {th }}$ JECFA meeting, which was held in 1973, whereas the specifications in the USA (through the FDA), the EU, and Japan were established after 1995. We considered that the hydroxyl value for Polysorbate 60 in the JECFA specification is higher than that in the EU, whereas the saponification value is lower. This is because of the differences in amount of impurities (e.g., free sorbitol-derived cyclic ethers) present in different Polysorbate 60 samples. Accordingly, we believe that the purities of newer commercial Polysorbate 60 samples are higher than those of older products, which were available when the JECFA specifications were discussed (around 1973), although we could not investigate the purities of the compounds available at that time.

The results indicate that the quality of the Polysorbate 60 products that are currently available worldwide is not consistent with the JECFA specifications for this additive in terms of the hydroxyl and saponification values. However, these products do fulfill the quality requirements in the USA, the EU, and Japan. Because the JECFA specifications are very important for a fair trade, according to the Agreement on Technical Barriers to Trade (TBT) and the Agreement on the Application of Sanitary and Phytosanitary Measures (SPS), these inconsistencies between the values determined for the studied Polysorbate 60 products and the JECFA specifications might induce serious trade disputes. In addition, according to the Agreements on TBT and SPS, if an international dispute happens, judgment will most likely be given based on an international standard.

Therefore, a revision of the current JECFA specifications for the hydroxyl and saponification values of Polysorbate 60 would be necessary for the international trade of current commercial products.

\section{Conclusion}

We investigated the hydroxyl and saponification values of Polysorbate 60 samples that are currently available worldwide as commercial food additives. Among the studied 27 samples, the hydroxyl values of 19 samples and the saponification values of 11 samples were observed to be outside the range specified by the JECFA. The results revealed that the JECFA specifications for Polysorbate 60 do not reflect the quality of the present samples. We would like to suggest a re-evaluation of the hydroxyl and saponification values of the current JECFA specifications for Polysorbate 60.

\section{Acknowledgements}

This work was partly supported by a Grant-in-Aid for Scientific Research from the Ministry of Health, Welfare, and Labor of Japan. The authors are deeply grateful to Dr. T. Hirakawa (International Life Science Institute, Japan), Dr. K. Seguro (Japan Food Additives Association), Mr. M. Yagi (Shin-Etsu Chemical Co. Ltd.), Dr. K. Asakura, Mr. H. Fujii (NOF Corporation), Mr. K. Miura (Yuka Sangyo Co. Ltd.), and Mr. T. Kobayashi (Oil \& Fat Industry Kaikan) for their valuable comments in preparation of this paper.

\section{REFERENCES}

[1] R. C. Rowe, P. J. Sheskey and P. J. Weller, "Handbook of Pharmaceutical Excipients,” Pharmaceutical Press, London, 2003, pp. 479-483, 591-595. 
[2] H. Vu. Dang, A. I. Gray, D. Watson, C. D. Bates, P. Scholes and G. M. Eccleston, "Composition Analysis of Two Batches of Polysorbate 60 Using MS and NMR Techniques," Journal of Pharmaceutical and Biomedical Analysis, Vol. 40, No. 5, 2006, pp. 1155-1165. http://dx.doi.org/10.1016/j.jpba.2005.10.007

[3] W. Xu, A. Nikolov, D. T. Wasan, A. Gonsalves and R. P. Borwanker, "Fat Particle Structure and Stability of Food Emulsions," Journal of Food Science, Vol. 63, No. 2, 1998, pp. 183-188.

http://dx.doi.org/10.1111/j.1365-2621.1998.tb15705.x
[4] N. H. Nash and L. M. Brickman, "Food EmulsifiersScience and Art," Journal of the American Oil Chemists' Society, Vol. 49, No. 8, 1972, pp. 457-461. http://dx.doi.org/10.1007/BF02582479

[5] G. Yilmazer and J. L. Kokini, "Effect of Polysorbate-60 on the Stability of O/W Emulsions Stabilized by Propylene Glycol Alginate and Xanthan Gum,” Journal of Texture Studies, Vol. 22, No. 3, 1991, pp. 289-301. http://dx.doi.org/10.1111/j.1745-4603.1991.tb00021.x 